
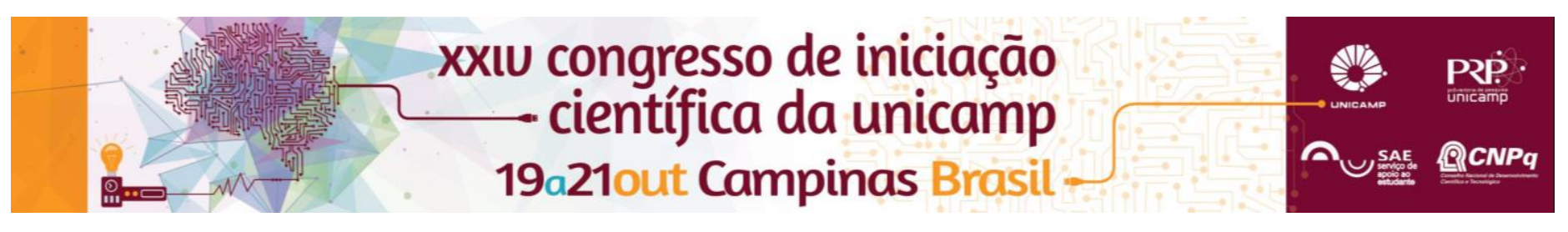

\title{
DTI analysis of temporal stem after anterior lobectomy
}

\author{
Pamela C. Pereira*, João P.S. Souza, Brunno M. Campos, Fernando Cendes, Helder Tedeschi, Enrico Ghizoni.
}

\begin{abstract}
This present work consists on a study of temporal stem (TS) components after modified anterior temporal lobectomy (MAL) in patients with temporal lobe epilepsy (TLE). The aim of DTI analysis of TS is to evaluate the anatomical consequences of this non selective access to the integrity of white matter fibers. Despite MAL has been proposed as a selective approach to spare the temporal stem and its fibers we could find some anatomic damage to the anterior part of the TS.
\end{abstract}

\section{Key words:}

epilepsy, tractography, lobectomy

\section{Introduction}

Temporal lobe epilepsy (TLE) is the most common type of focal epilepsy in adults. $11 \%$ to $42 \%$ of patients with TLE have high refractory to the use of anti-epileptic drugs. The current treatment of choice for those patients is surgical resection of the mesial temporal region of structures (amygdala and hippocampus).

In the surgical access path for mesial temporal region is the temporal stem (TS). The major fiber tracts in the TS are uncinate (FU), lower occipitofrontal issue (FOI) and optic radiation (OR). As these fibers are located in the anterior portion of TS are more susceptible to damage in epilepsy surgery, with major clinical implications. In Epilepsy Surgery Division of University of Campinas was developed modified anterior temporal lobectomy (MALT). Tractography uses DTI to create an anatomical map in vivo.

The aim of DTI analysis of TS is to evaluate the anatomical consequences of this non selective access to the integrity of white matter fibers.

\section{Results and Discussion}

To the study we formed two groups: 26 patients who underwent modified anterior temporal lobectomy from 2010 to 2014 (MAL group); 33 healthy controls (control group - CTR). DTI analysis was done in both groups and the tracts analyzed were inferior fronto - occipital fasciculus (IFOF), uncinate fasciculus (UF) and optic radiation (OR) of TS. We analyzed if the tracts showed disruption and fraction of anisotropy (FA) which measures de direction of anisotropy, the result is from 0 (isotropic movement) to 1 (anisotropic movement); low numbers of FA indicates destruction of fibers.

This study found that TS analysis showed complete interruption of UF in $80,77 \%$ of MAL patients and $11,54 \%$ presented complete interruption of the IFOF. Utilizing controls, there was no significant difference comparing the fractional anisotropy (FA) from OR of CTR and MAL groups, while the comparisons of the UF and IFOF remaining fibers between the MAL and CTR groups showed significant difference $(p<0,05)$ with lower FA for the surgical group.

The results found an important damage to the UF and IFOF fibers that are located at the anterior portion of the TS and implication on the integrity of remaining fibers. The most anterior fibers of the OR showed to be severed in some patients but the remaining fibers didn't show to be damaged. This suggests also involvement of function, specially of IFOF and UF which had lowers FA.

Image: Postoperative magnetic resonance image after the modified anterior temporal lobectomy and tractography of the inferior occipital fasciculus
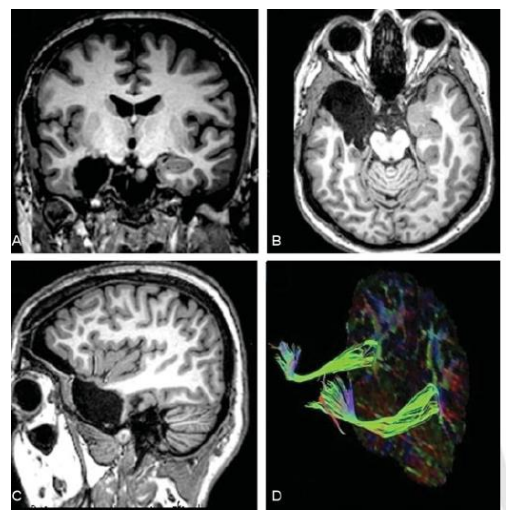

\section{Conclusions}

Despite MAL has been proposed as a selective approach to spare the temporal stem and its fibers we could find some anatomic damage to the anterior part of the TS, mainly the UF and IFOF. Further correlations between these findings and visual field exams and neuropsychological data are needed for better understanding the clinical value of these DTI abnormalities, allowing better understanding of the implications of the MAL in patients's quality of life.

\section{Acknowledgement}

grant 2015/10331-0, São Paulo Research Foundation (FAPESP)

\footnotetext{
${ }^{1}$ Enrico Ghizoni Joao Paulo Almeida; Andrei F. Joaquim; Clarissa L. Yasuda; Brunno M. de Campos; Helder Tedeschi; Fernando Cendes - Modified Anterior Temporal Lobectomy: Anatomical Landmarks and Operative Technique -Journal of Neurological Surgery_Part A - 2015
} 